\title{
Infection Dynamics in Viral Spread and Interference Under the Synergism Between Cucumber mosaic virus and Turnip mosaic virus
}

\author{
Minoru Takeshita, ${ }^{1}$ Emiko Koizumi, ${ }^{1}$ Makiko Noguchi, ${ }^{1}$ Kae Sueda, ${ }^{2}$ Hanako Shimura, ${ }^{2}$ Noriko Ishikawa, ${ }^{3}$ \\ Hideyuki Matsuura, ${ }^{2}$ Kazusato Ohshima, ${ }^{4}$ Tomohide Natsuaki, ${ }^{3}$ Shigeru Kuwata, ${ }^{5}$ Naruto Furuya, ${ }^{1}$ \\ Kenichi Tsuchiya, ${ }^{1}$ and Chikara Masuta ${ }^{2}$ \\ ${ }^{1}$ Laboratory of Plant Pathology, Faculty of Agriculture, Kyushu University, 6-10-1 Hakozaki, Higashi-ku, Fukuoka 812-8581, \\ Japan; ${ }^{2}$ Graduate School of Agriculture, Hokkaido University, Kita-ku kita 9, Nishi 9, Sapporo 060-8589, Japan; ${ }^{3}$ Faculty of \\ Agriculture, Utsunomiya University, Mine-machi 350, Utsunomiya 321-8505, Japan; ${ }^{4}$ Faculty of Agriculture, Saga University, \\ Saga 840-8502, Japan; ${ }^{5}$ School of Agriculture, Meiji University, Tama-ku, Kawasaki 214-8571, Japan
}

Submitted 18 June 2011. Accepted 25 August 2011.

\begin{abstract}
Mixed infection of Cucumber mosaic virus (CMV) and Turnip mosaic virus (TuMV) induced more severe symptoms on Nicotiana benthamiana than single infection. To dissect the relationships between spatial infection patterns and the $2 \mathrm{~b}$ protein (2b) of CMV in single or mixed infections, the CMV vectors expressing enhanced green fluorescent or Discosoma sp. red fluorescent proteins (EGFP [EG] or DsRed2 [Ds], respectively) were constructed from the same wild-type CMVY and used for inoculation onto $N$. benthamiana. CMV2-A1 vector (C2-A1 [A1]) has a functional $2 \mathrm{~b}$ while CMV-H1 vector (C2-H1 [H1]) is 2b deficient. As we expected from the $2 \mathrm{~b}$ function as an RNA silencing suppressor (RSS), in a single infection, A1Ds retained a high level of accumulation at initial infection sites and showed extensive fluorescence in upper, noninoculated leaves, whereas H1Ds disappeared rapidly at initial infection sites and could not spread efficiently in upper, noninoculated leaf tissues. In various mixed infections, we found two phenomena providing novel insights into the relationships among RSS, viral synergism, and interference. First, H1Ds could not spread efficiently from vasculature into nonvascular tissues with or without TuMV, suggesting that RNA silencing was not involved in CMV unloading from vasculature. These results indicated that $2 \mathrm{~b}$ could promote $\mathrm{CMV}$ to unload from vasculature into nonvascular tissues, and that this $2 \mathrm{~b}$ function might be independent of its RSS activity. Second, we detected spatial interference (local interference) between A1Ds and A1EG in mixed infection with TuMV, between A1Ds (or H1Ds) and TuMV, and between H1Ds and H1EG. This observation suggested that local interference between two viruses was established even in the synergism between CMV and TuMV and, again, RNA silencing did not seem to contribute greatly to this phenomenon.
\end{abstract}

Plant viral RNA silencing suppressors (RSS) have been suggested to define tissue invasion patterns in various host plant-

Corresponding co-authors: M. Takeshita; Telephone: +81 92 6422836; Fax: +81 92 6422836; E-mail: minorutk@agr.kyushu-u.ac.jp; and C. Masuta; Telephone: +81 11 7062807; Fax: +81 11 7062483; E-mail: masuta@ res.agr.hokudai.ac.jp

* The $\boldsymbol{e}$-Xtra logo stands for "electronic extra" and indicates that eleven supplementary figures and one supplementary table are published online. virus combinations. Voinnet and associates (1999) showed that virus-encoded RSS have distinct modes of action in the host plant gene-silencing machinery, which is well known as a host defense mechanism against viral infection.

It also has been suggested that viral RSS have important roles in tissue invasion patterns in mixed virus infections. Coinfection of two or more different plant viruses can exacerbate symptom severity and increase the titer of the viruses. The symbiotic interaction is well known as synergism and can be seen most often in interactions between a Potyvirus sp. and a virus in a different family; for example, Turnip mosaic virus (TuMV) and Cucumber mosaic virus (CMV) (Sano and Kojima 1989; Takeshita and Takanami 2000) and Potato virus Y (PVY) and Potato virus X (PVX) (Brigneti et al. 1998; González-Jara et al. 2004). The helper component proteinase (HC-Pro) gene encoded by potyviruses, which is a strong RSS, plays a key role in such synergistic interactions (Brigneti et al. 1998).

One of the best characterized RSS, the $2 b$ protein $(2 b)$ of CMV (Palukaitis and García-Arenal 2003), was discovered as encoded by a small gene which overlaps, in part, with the $3^{\prime}$ end of the $2 \mathrm{a}$ gene of CMV (Ding et al. 1994). The $2 \mathrm{~b}$ protein has complex activities to suppress RNA silencing, control host basal resistances, and operate synergistic interactions with Potyvirus spp. in both a virus- and a host-specific manner (Brigneti et al. 1998; Ding et al. 1994, 1995; Ji and Ding 2001; Mascia et al. 2010; Ryang et al. 2004, 2005; Shi et al. 2003; Soards et al. 2002; Wang et al. 2004). The 2b protein can inhibit systemic spread of transgene silencing into newly developing leaves, although it does not suppress the RNA-silencing machinery established in plant tissues before virus invasion (Brigneti et al. 1998). The $2 \mathrm{~b}$ protein can bind to Argonaute 1 and small RNAs, suggesting its roles in preventing amplification and spread of short-interfering RNAs (siRNAs) which are produced by the RNA silencing machinery (Goto et al. 2007; Zhang et al. 2006). Most interestingly, González and associates (2010) suggested that the ability of $2 b$ to bind small RNAs is critical for the RSS activity.

Dynamic functions of $2 b$ in single and mixed infections, however, still remain to be elucidated in great detail because of lack of a virus vector system to monitor the infection dynamics of CMV throughout plant tissues. In the previous work by Otagaki and associates (2006), a novel virus vector was developed using the biologically active cDNA clone of CMV-Y RNA 2 (Suzuki et al. 1991). The vector, designated CMV2-A1 
(C2-A1), retained functions required for efficient systemic infection of CMV in Nicotiana benthamiana. Subsequently, Matsuo and associates (2007) constructed another CMV-Y RNA2-based vector (CMV-H1 [C2-H1]) to produce foreign proteins, by replacement of the entire open reading frame (ORF) of the $2 b$ gene. In the present work, we established a new fluorescent-protein-expressing CMV vector system based on $\mathrm{C} 2-\mathrm{A} 1$ and $\mathrm{C} 2-\mathrm{H} 1$ which enables us to visualize the infection dynamics of CMV.

The aim of this study is to elucidate the viral infection dynamics of CMV directed by $2 b$ functions. Using spatial analyses, we adopted the CMV vector system to monitor single infections, and applied a CMV/TuMV vector system to dissect mixed infections in synergism, respectively. In single infections, the CMV vector system successfully visualized the complex activities of $2 \mathrm{~b}$ during viral infection dynamics. In mixed infections, the CMV/TuMV vector system first revealed the complementary function of $2 b$ to unload CMV from vascular tissues and the spatial competition between CMV and TuMV under synergism.

\section{RESULTS}

Generation of fluorescence-expressing CMV and TuMV.

The C2-A1 vector (A1) has the 5' two-thirds of the $2 \mathrm{~b}$ gene while the $\mathrm{C} 2-\mathrm{H} 1$ (H1) lacked the entire $2 \mathrm{~b}$ gene (Fig. 1) (Otagaki et al. 2006; Matsuo et al. 2007). The RSS activity of the truncated $2 \mathrm{~b}$ in A1 was found to be almost equal to that of the authentic $2 \mathrm{~b}$ in $N$. benthamiana protoplasts (Supplementary Fig. 1). We inserted either the Discosoma sp. red fluorescent protein (DsRed2) or enhanced green fluorescent protein (EGFP) gene into A1 in fusion with the $2 \mathrm{~b}$ gene to create $\mathrm{C} 2-$ A1-DsRed2 (A1Ds) and C2-A1-EGFP (A1EG), respectively. Likewise, the $\mathrm{H} 1$ constructs containing the DsRed 2 and EGFP gene were named C2-H1-DsRed2 (H1Ds) and C2-H1-EGFP (H1EG), respectively. In vitro transcripts of those constructs were inoculated with RNAs 1 and 3 of CMV-Y onto N. benthamiana to create the mutated viruses named $\mathrm{C} 2-\mathrm{A} 1, \mathrm{C} 2-\mathrm{H} 1$, A1Ds, A1EG, H1Ds, and H1EG for convenience. The fluores- cence-expressing, recombinant CMV spread systemically and were stable at least for 12 days postinoculation (dpi). However, when we used sap from the infected tissues as an inoculum (second passage), the plants sometimes showed reduced fluorescence in systemically infected leaves due to spontaneous deletions in the DsRed2 and EGFP genes (data not shown). Thus, we always used in vitro transcripts for inoculation to monitor the virus spread so that the viruses would not lose fluorescence expression by serial passages. For TuMV, we used the TuMV vector expressing the EGFP gene (TuEG) (Supplementary Fig. 2) (Suehiro et al. 2004).

\section{Nature of viral spread of fluorescence-expressing CMV and TuMV in a single infection.}

To visualize the $2 \mathrm{~b}$-directed infection dynamics of CMV, the fluorescence-expressing CMV were inoculated onto $N$. benthamiana. A1Ds (or A1EG) formed distinct fluorescent foci at the initial infection sites and displayed radial spread of fluorescence throughout the upper, noninoculated leaf tissues (Fig. 2A for A1Ds and data not shown for A1EG). As expected, the plants infected with A1Ds (or A1EG) developed systemic chlorosis or yellow mosaics in the upper, noninoculated leaves with stunting, which were similar to those induced by CMV-Y and C2-A1 for A1Ds and data not shown for A1EG). In contrast, although the plants infected with H1Ds (or H1EG) developed similar fluorescent foci in the inoculated leaves to those induced by A1Ds, the intensity of the fluorescence at the center of those foci rapidly decreased by 7 dpi (Fig. 2A for H1Ds and data not shown for H1EG). In the upper, noninoculated leaves, the fluorescent signals were mainly localized in the veins and, thus, dark zones along the veins were observable over the leaves (Fig. 2A for H1Ds). Plants infected with H1Ds (or H1EG) showed moderate development of mild vein clearing, systemically faint mosaic, and slight stunting, which were similar to those induced by C2H1 (Supplementary Fig. 3A for C2-H1, and data not shown for H1Ds and H1EG). The results from A1Ds (or A1EG) relative to H1Ds (or H1EG) indicated that the $2 b$ retarded activation of both locally and systemically antiviral pathways in the processes of viral cell-to-cell and vascular movement.

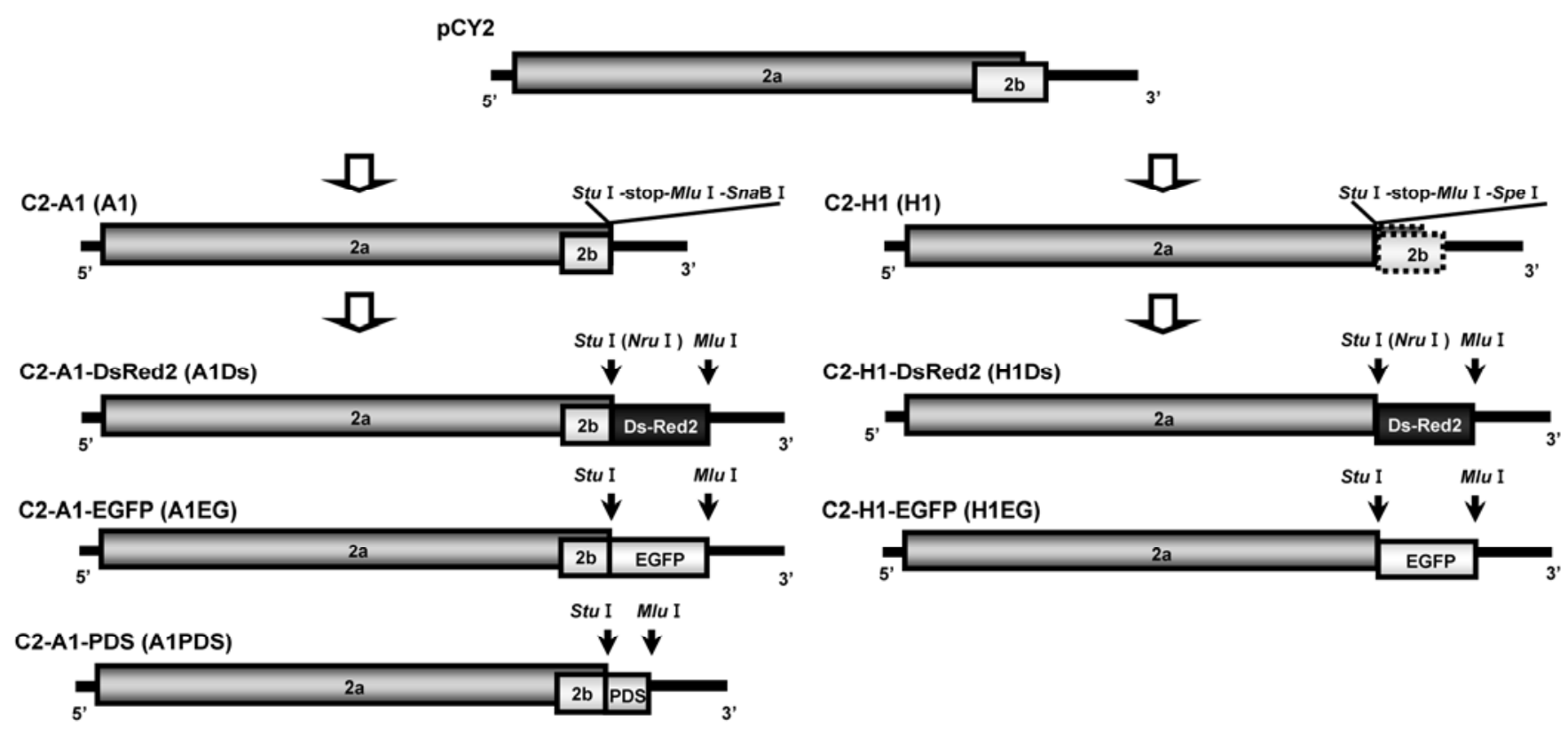

Fig. 1. Schematic representation of the modified Cucumber mosaic virus (CMV) vectors. C2-A1 type vectors: the Discosoma sp. red fluorescent protein (DsRed2)- and enhanced green fluorescent protein (EGFP)-expressing vectors and the phytoene desaturase (PDS) gene-silencing vector were developed on the basis of the C2-A1 vector. C2-H1 type vectors: the DsRed2- and the EGFP-expressing vectors were constructed on the basis of the C2-H1 vector. Restriction sites used for insertion of the gene fragments are indicated by black arrows. 
In leaves inoculated with TuEG, radial spread of fluorescent signals developed from each infection site, and the virus spread systemically (Fig. 2A). TuEG also induced mosaic symptoms with stunting similar to those induced by TuMV.

\section{The 2b can help CMV unload from leaf veins.}

To investigate whether $2 \mathrm{~b}$ is involved in the synergism between CMV and TuMV, we simultaneously inoculated C2-A1 (or C2-H1)/TuMV onto $N$. benthamiana. Both C2-A1/TuMVand $\mathrm{C} 2-\mathrm{H} 1 / \mathrm{TuMV}$-infected plants showed systemic symptoms, with severe yellowing and stunting followed by lethal necrosis, similar to the symptoms caused by CMV-Y/TuMV and never observed in the plants infected with each virus alone. We confirmed the systemic infection of CMV-Y, C2-A1, C2-H1, and TuMV by reverse-transcription polymerase chain reaction (RTPCR) (data not shown). These results indicated that $2 \mathrm{~b}$ was not important for the synergistically severe symptoms induced by the mixed infection of $\mathrm{CMV}$ and TuMV. To monitor viral spread in the synergism, we inoculated A1Ds (or H1Ds) with TuMV, and obtained similar results (Supplementary Fig. 4 for H1Ds/TuMV, and data not shown for A1Ds/TuMV ). In the A1Ds/TuMV-infected plants, we observed uniform distribution of A1Ds over all the infected leaf tissues (Fig. 2B). However, when H1Ds was inoculated together with TuMV, viral fluorescence was detected mainly in the vascular tissues (Fig. 2B), suggesting that $2 \mathrm{~b}$ was important for the virus to unload from the veins. To confirm 2b's role in viral unloading, we created transgenic $N$. benthamiana plants expressing the GFP-tagged $2 \mathrm{~b}(2 \mathrm{~b}-\mathrm{GFP} \mathrm{Nb})$, the RSS activity of which enhanced accumulation of $\mathrm{C} 2-\mathrm{H} 1$ to a level equivalent to that of $\mathrm{C} 2-\mathrm{A} 1$ in wildtype $N$. benthamiana plants (data not shown). When 2b-GFP $\mathrm{Nb}$ plants were inoculated with H1Ds, we observed that the mesophyll cells showed a more uniform distribution of fluorescence in the upper, noninoculated leaves and, thus, confirmed complementation of viral unloading by $2 b$ (Fig. 2B). Furthermore, when $2 \mathrm{~b}$-GFP $\mathrm{Nb}$ plants were inoculated with H1Ds/
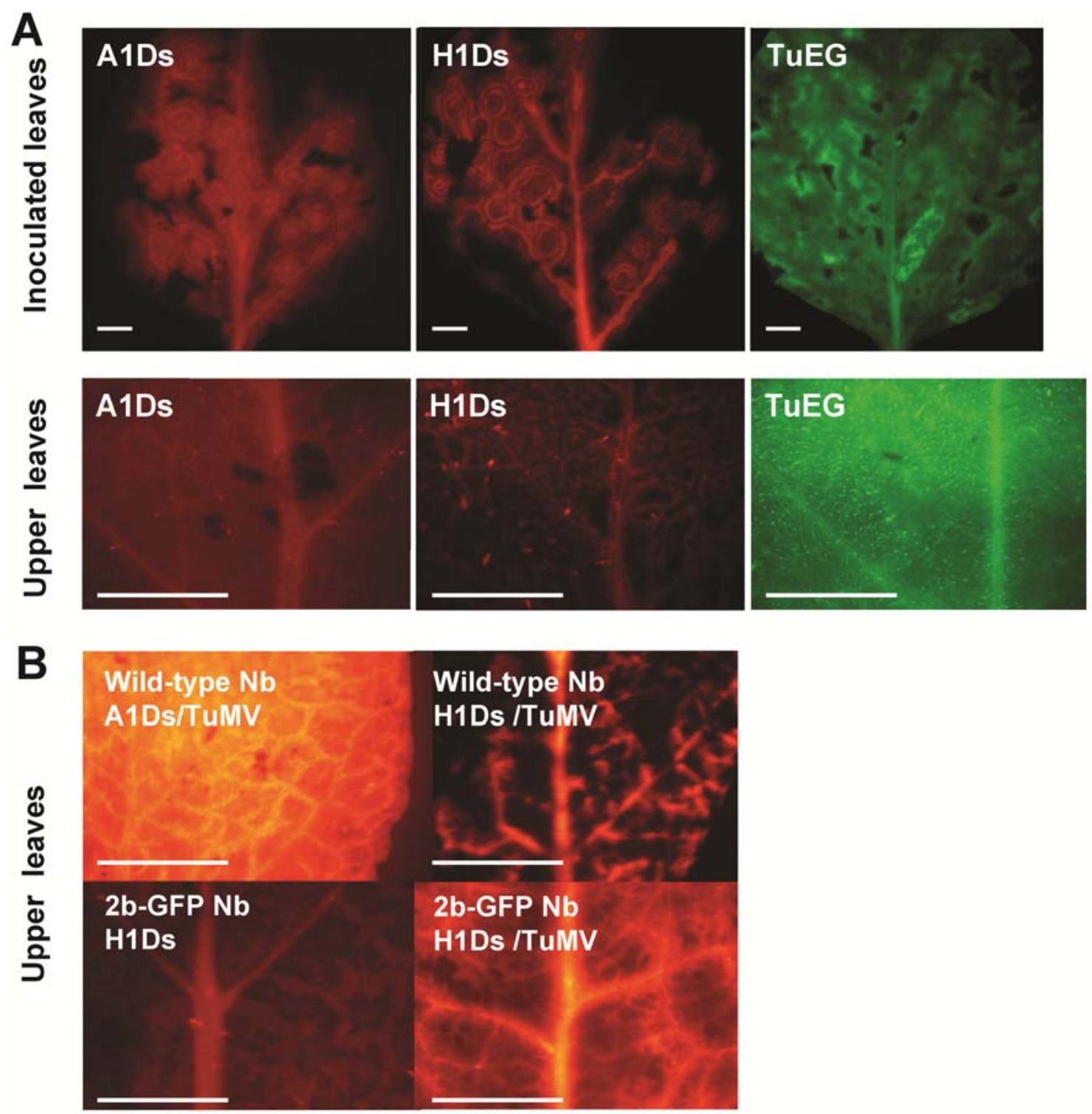

Fig. 2. The $2 \mathrm{~b}$ protein-directed infection dynamics of Cucumber mosaic virus (CMV) in single and mixed infections with Turnip mosaic virus (TuMV). A1Ds and A1EG indicate CMV-Y mutants which encode the 5' two-thirds of the $2 \mathrm{~b}$ gene fused to the Discosoma sp. red fluorescent protein (DsRed2) gene and to the enhanced green fluorescent protein (EGFP) gene, respectively. TuEG indicates a mutated TuMV expressing the EGFP gene. A, Cell-to-cell movement of A1Ds, H1Ds, and TuEG in the inoculated leaves at 7 days postinoculation (dpi), and systemic spread of the viruses in the noninoculated third upper leaves at 9 dpi in wild-type Nicotiana benthamiana. B, Systemic spread of A1Ds and H1Ds in single and mixed infections with TuMV in the noninoculated third upper leaves at $8 \mathrm{dpi}$ in wild-type $N$. benthamiana (Wild-type $\mathrm{Nb}$ ) and the 2b-GFP-expressing transgenic $N$. benthamiana (2b-GFP Nb). Scale bars represent $4 \mathrm{~mm}$. 
TuMV, the observed fluorescence in the upper, noninoculated leaves was markedly enhanced by the synergism to the extent observed in A1Ds/TuMV-infected leaves; the veins were still heavily fluorescent (Fig. 2B). The H1Ds-infected 2b-GFP Nb plants displayed systemic chlorosis or yellow mosaic symptoms which were more severe than those induced in the H1Dsinfected wild-type $N$. benthamiana plants. This confirmed that $2 \mathrm{~b}$ had complementary functions to increase symptom severity. On the other hand, the TuMV-infected 2b-GFP Nb plants did not show increased symptom severity in comparison with those on the TuMV-infected wild-type $N$. benthamiana plants, suggesting that $2 \mathrm{~b}$ alone did not play important roles in formation of synergistic symptoms in mixed infections with TuMV. The H1Ds/TuMV-infected 2b-GFP Nb plants developed severe systemic mosaics with stunting which were similar to those on the H1Ds/TuMV-infected wild-type $N$. benthamiana plants. The results again suggested that the synergistic symptoms could be caused by excessive accumulation of CMV in leaf veins. These results all indicated that $2 b$ has a defined role in promoting viral unloading from leaf veins in the upper, noninoculated leaves.

\section{Identification of "local interference" in the synergism between two viruses.}

In our experimental system, we first confirmed synergistic interactions between A1Ds (or H1Ds) and TuMV and between CMV and TuMV (Fig. 2B). To monitor viral spread, we simultaneously inoculated A1Ds (or H1Ds)/TuEG onto $N$. benthamiana and followed the fluorescence from the recombinant viruses.
The A1Ds (or H1Ds)/TuEG-inoculated plants developed severe systemic mosaic and stunting followed by lethal systemic necrosis, which were equivalent to those produced on the CMVY/TuMV-inoculated plants (data not shown for H1Ds/TuEG). Although mixed fluorescent signals from two viruses certainly were detected, we found that the viruses spread radially from their infection sites in the inoculated leaves but often not into the same cells (Fig. 3A for A1Ds/TuEG and Supplementary Fig. 5A for H1Ds/TuEG). This suggested that spatial interference occurred between CMV and TuMV at an early stage of mixed infection of the two viruses. We have named this phenomenon "local interference". According to the observations made using a confocal laser-scanning microscope, local interference was not found in the multiple layers of cells in the sites where mixed fluorescent signals from two viruses were detected by fluorescence microscopy (data not shown). Interestingly, the local interference between A1Ds (or H1Ds) and TuEG seemed to cause a delay of the systemic movement of A1Ds (or H1Ds), approximately until $7 \mathrm{dpi}$ (Fig. 3B for A1Ds/TuEG). In the serial inoculations with A1Ds 2 days after TuEG (TuEG-A1Ds) or TuEG 2 days after A1Ds (A1Ds-TuEG), local interference also was observed in the inoculated leaves (Fig. 4A for TuEG-A1Ds and Supplementary Fig. 6A for A1Ds-TuEG). In the TuEGA1Ds inoculation, radial spread of A1Ds was significantly restricted by prior infection of TuEG (Fig. 4A). This also occurred in the cell-to-cell movement of TuEG in the A1Ds-TuEG inoculation. Delays of systemic movement of A1Ds and TuEG were observed in the TuEG-A1Ds and A1Ds-TuEG inoculations, respectively (Fig. 4B for TuEG-A1Ds).
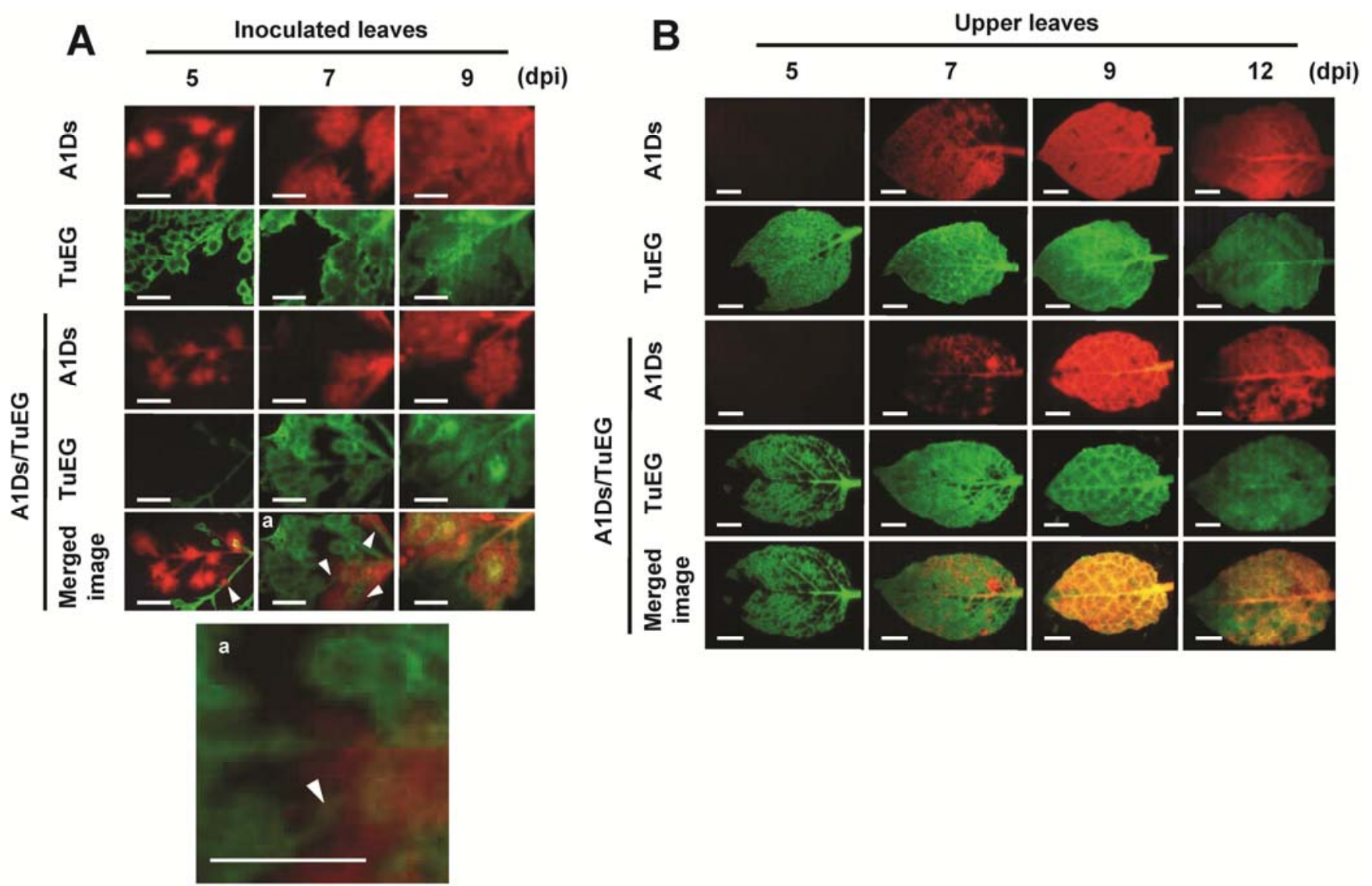

Fig. 3. Local interference and synergistic interactions between Cucumber mosaic virus A1 vector expressing the Discosoma sp. red fluorescent protein (DsRed2) gene (A1Ds) and Turnip mosaic virus vector expressing the enhanced green fluorescent protein gene (TuEG) in simultaneous infections. A, Cellto-cell movement of A1Ds or TuEG in single infections and that of A1Ds/TuEG in simultaneous infections in the inoculated leaf tissues at 5, 7, and 9 days postinoculation (dpi). White arrows indicate the sites of local interference between A1Ds and TuEG. a, A white arrow in the magnification indicates an example for local interference. B, Systemic spread of A1Ds or TuEG in single infections and that of A1Ds/TuEG in simultaneous infections in the noninoculated fourth upper leaves at 5, 7, 9, and $12 \mathrm{dpi}$. Scale bars represent $4 \mathrm{~mm}$. 
To understand which virus benefits from mixed infection of A1Ds and TuEG, we analyzed virus accumulation in the synergy between A1Ds and TuEG by RT quantitative (q)PCR. The accumulation levels of A1Ds in the A1Ds/TuEG-inoculated plants were significantly increased but, inversely, the accumulation of TuEG was decreased below $50 \%$ of the single infection control at $8 \mathrm{dpi}$ (Fig. 5A and B). In addition, semiquantitative RT-PCR demonstrated that the accumulation level of H1Ds was elevated in the mixed infection, whereas TuEG was not affected by H1Ds but decreased to a certain degree by A1Ds when measured at 10 dpi (Supplementary Fig. 7).

Taken together, the results might reflect the consequence of local interference between CMV and TuMV (i.e., the accumulation of TuMV was preferentially restricted by far more efficient CMV multiplication). Therefore, we found here that two adverse events of interference and synergism co-existed together in the mixed infection of CMV and TuMV.

\section{Neither $2 \mathrm{~b}$ nor low-temperature treatment altered} local interference between two viruses.

Because RNA silencing is thought to be involved in the viral interference phenomenon called cross-protection and also in synergism (at least partially), we also investigated the role of the CMV 2b RSS in the observed local interference. Local interference was confirmed between A1Ds and A1EG that were simultaneously or serially inoculated (Supplementary Figs. 8 and 9). When the two CMV constructs (A1Ds and A1EG) and TuMV were simultaneously inoculated, we could still identify local interference between two CMV (A1Ds and A1EG), suggesting that both RSS, CMV 2b and TuMV HCPro, did not prevent the local interference (Fig. 6A and B). In agreement with this, we also observed very distinct local interference between two 2b-deficient CMV in the plants infected with H1Ds/H1EG (Supplementary Fig. 10). Thus, these results indicated that RNA silencing might not be involved in the local interference.

Because virus-induced gene silencing (VIGS) is supposed to be inhibited at low temperature, according to Szittya and associates (2003), we examined whether low-temperature treatment $\left(15^{\circ} \mathrm{C}\right)$ affects the local interference between A1Ds and A1EG in mixed infection with TuMV. Although the plants developed no synergistic severe symptoms at $15^{\circ} \mathrm{C}$ for $21 \mathrm{dpi}$ (data not shown), we could clearly identify local interference, which also could be observed in the plants co-inoculated with A1Ds and A1EG (Fig. 6). Similarly, in a parallel check at $15^{\circ} \mathrm{C}$, the A1PDS construct against the endogenous phytoene desaturase (PDS) gene (Fig. 1) was confirmed not to induce VIGS even at $21 \mathrm{dpi}$, because we did not observe photobleaching that was clearly induced at 25 and $20^{\circ} \mathrm{C}$ (day and night, respectively) by 14 dpi (Supplementary Fig. 11). The results
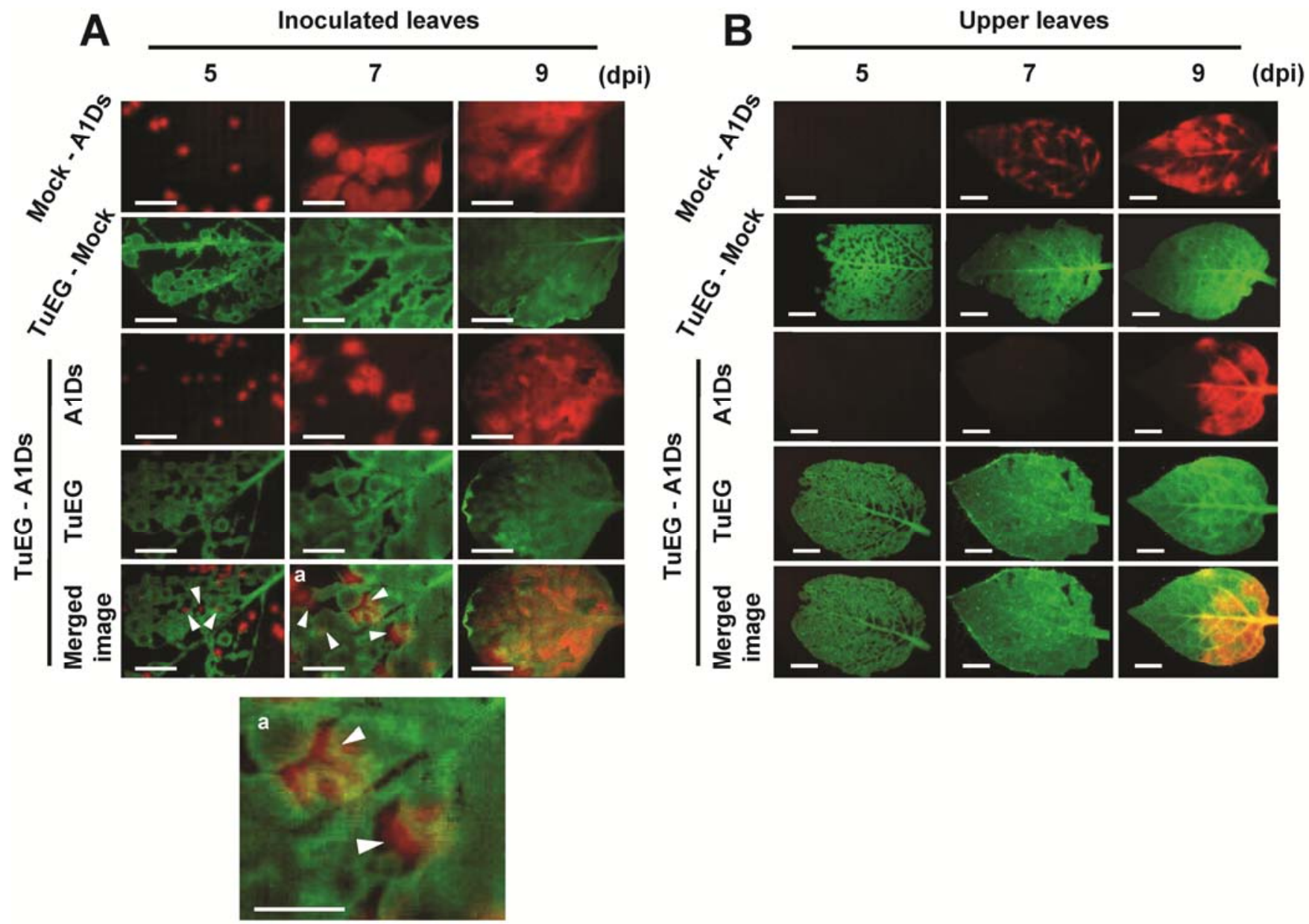

Fig. 4. Local interference and subsequent synergy between Turnip mosaic virus vector expressing the enhanced green fluorescent protein gene (TuEG) and Cucumber mosaic virus A1 vector expressing the Discosoma sp. red fluorescent protein (A1Ds) in serial infections. A, Cell-to-cell movement of A1Ds after mock inoculation and that of TuEG before mock inoculation in single infections, and that of A1Ds and TuEG in serial infections of A1Ds 2 days after TuEG in the inoculated leaf tissues at 5, 7, and 9 days postinoculation (dpi). White arrows indicate the sites of local interference between TuEG and A1Ds. a, White arrows in the magnification indicate examples for local interference. B, Systemic spread of A1Ds after mock inoculation and TuEG before mock inoculation in single infections, and that of TuEG and A1Ds in serial infections of A1Ds after TuEG in the noninoculated fourth upper leaves at 5, 7, and 9 dpi. Scale bars represent $4 \mathrm{~mm}$. 
confirmed the successful attenuation of RNA silencing in the assay at $15^{\circ} \mathrm{C}$. This observation again suggested that RNA silencing was not important for the local interference, in agreement with the previous results on the role of the viral RSS.

\section{DISCUSSION}

In this study, we found undefined functions of the $2 \mathrm{~b}$ protein involved in the infection dynamics of CMV in single and in mixed infections with TuMV. The novel CMV/TuMV vector system also revealed local interference between CMV and TuMV.

A1-2b retarded activation

of antiviral responses at infection sites of CMV.

Goto and associates (2007) and González and associates (2010) previously reported that the ability of $2 \mathrm{~b}$ to bind small RNAs was critical for its RSS activity. Because the A1-2b has been demonstrated to have the same ability to bind small RNAs (Kanazawa et al. 2011), we expected to observe the
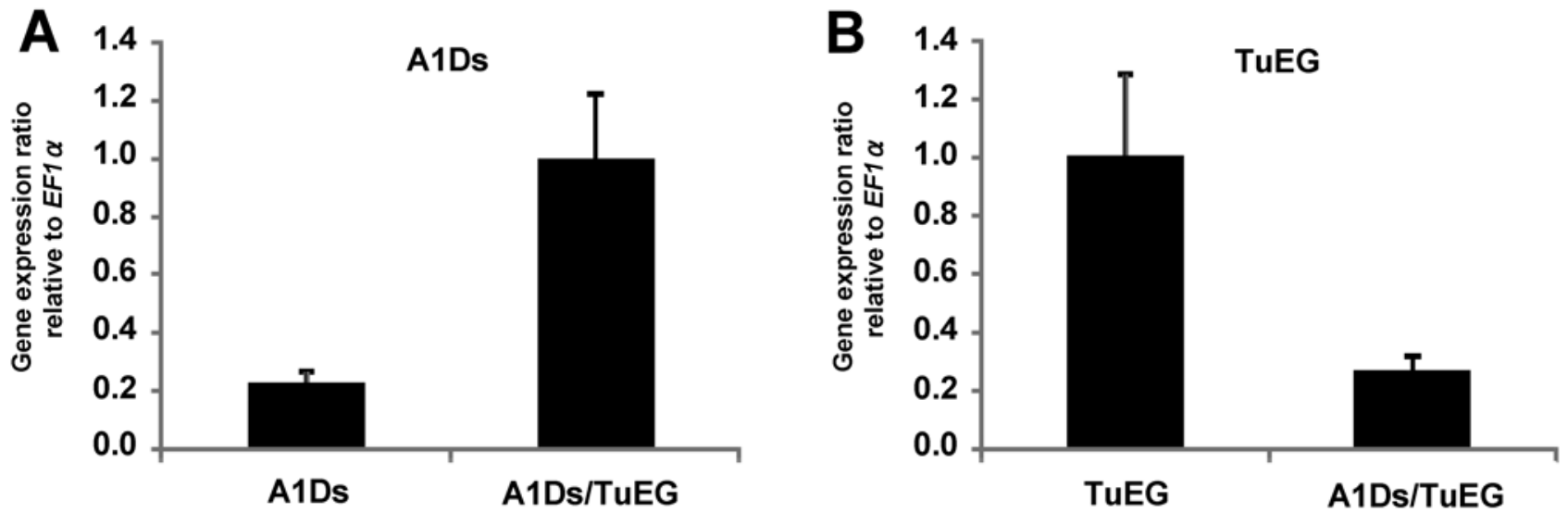

Fig. 5. Relative accumulation of Cucumber mosaic virus A1 vector expressing the Discosoma sp. red fluorescent protein (A1Ds) and Turnip mosaic virus vector expressing the enhanced green fluorescent protein gene (TuEG) in either singly or simultaneously infected Nicotiana benthamiana. Plants were maintained at 25 and $20^{\circ} \mathrm{C}$ (day and night, respectively). Systemically infected leaf tissues were harvested at 8 days postinoculation (dpi). Accumulation levels of A1Ds and TuEG were measured by real-time reverse-transcription polymerase chain reaction. Virus RNA levels (in arbitrary units normalized to $E F 1 \alpha$ ) with mean \pm standard errors. A, Relative accumulation of A1Ds in the plants inoculated with A1Ds or A1Ds/TuEG. B, Relative accumulation of TuEG in the plants inoculated with TuEG or A1Ds/TuEG.

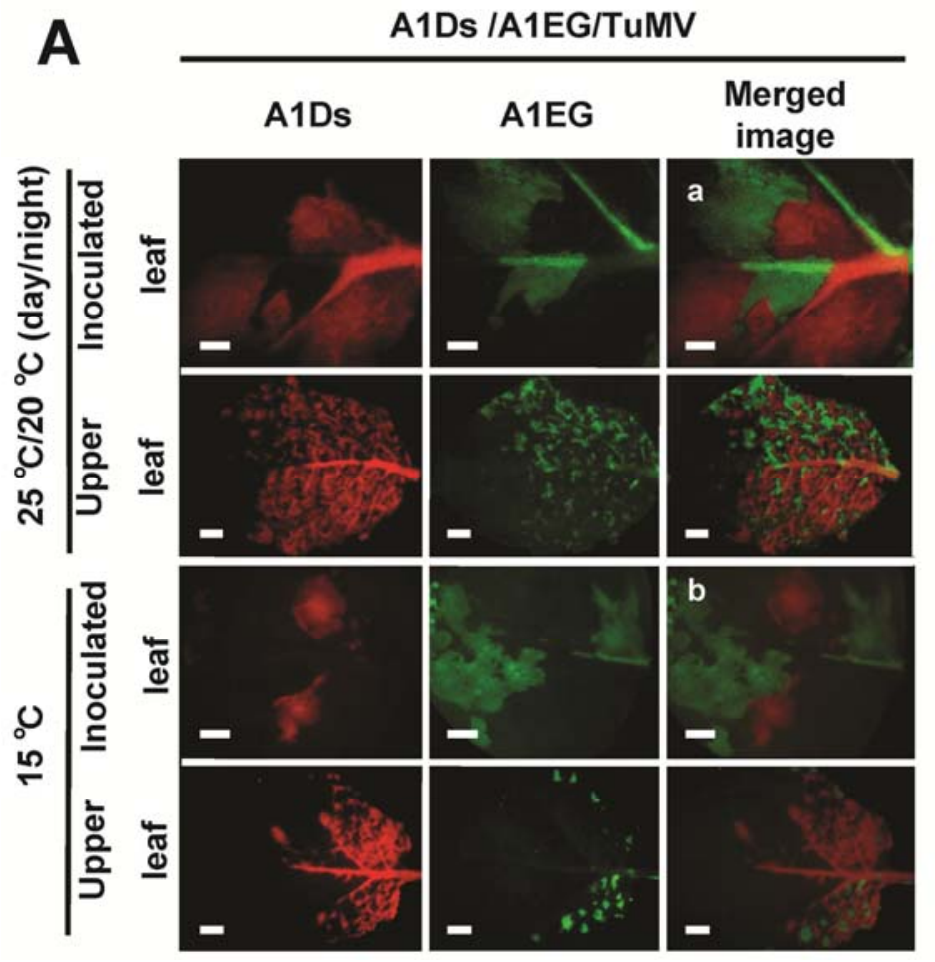

B

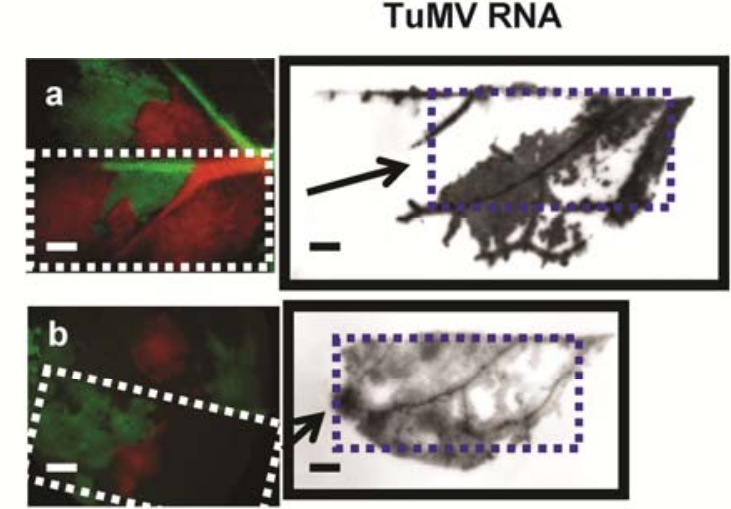

Fig. 6. Spatial competition between Cucumber mosaic virus A1 (A1) vector expressing the Discosoma sp. red fluorescent protein (A1Ds) and A1 enhanced green fluorescent protein (A1EG) in Nicotiana benthamiana triply infected with A1Ds/A1EG/Turnip mosaic virus (TuMV). A, Cell-to-cell movement and systemic spread of A1Ds and A1EG in triple infections with TuMV at 25 and $20^{\circ} \mathrm{C}$ (day and night, respectively) at 7 days postinoculation (dpi) and those at $15^{\circ} \mathrm{C}$ at 14 dpi. a and $\mathbf{~ b}$, Inoculated leaf tissues were further analyzed by press blot hybridization. B, Cell-to-cell spread of TuMV in the inoculated leaf tissues. $\mathbf{a}$ and $\mathbf{b}$, The same leaf tissues shown in A. Black arrows indicate the same regions surrounded by dotted lines that were analyzed by TuMV RNA-specific press blot hybridization after fluorescence microscopy observation. Black and gray zones indicate accumulation of TuMV RNA. Scale bars represent 4 mm. 
ability of A1-2b to interfere with RNA silencing as monitored by the efficiency of viral spread (fluorescence). In single infection, A1Ds and A1EG showed intensive fluorescence, which was quite uniform throughout the plant tissues, at least at the initial infection stage. In contrast, a rapid decrease in intensity of fluorescence was observed in the center of infection foci of H1Ds and H1EG and, subsequently, faint fluorescence with a mosaic pattern was formed in the upper, noninoculated leaves. This observation that the fluorescence of $\mathrm{A} 1$ was much stronger than $2 \mathrm{~b}$-deficient $\mathrm{H} 1$ indicates that $\mathrm{A} 1-2 \mathrm{~b}$ was certainly functional in delaying antiviral responses which might be involved in host RNA silencing.

\section{The $2 \mathrm{~b}$ has a new role in controlling spatial spread of CMV from leaf veins.}

In mixed infections with TuEG, we observed that A1Ds accumulated at a high level over all the leaf tissues in upper, noninoculated leaves, whereas H1Ds was preferentially localized in vascular tissues. In addition, the $2 \mathrm{~b}$-expressing transgenic plants actually promoted H1Ds to unload from vascular to nonvascular tissues. Therefore, these results suggested that $2 b$ had an undefined function to stimulate the virus to egress from veins in the upper, noninoculated leaves. Our single infection experiments indicated that $2 \mathrm{~b}$ could enhance viral movement to upper, noninoculated leaves and maintain stable accumulation of the virus. This could be essentially explained by the 2b's RSS activity, which protected viral genomic and subgenomic RNAs against host RNA silencing. However, in our mixed infection experiments, it was unlikely that RNA silencing played a major role in the mechanism that prevented H1Ds unloading from vascular tissues, because the strong RSS, TuMV HC-Pro, should also have diminished host RNA silencing in the mixed infection. Thus, we hypothesize that $2 \mathrm{~b}$ controls spatial spread of CMV from veins in upper, noninoculated leaves by a mechanism that is independent of suppression of RNA silencing. Efficient unloading of CMV from vascular tissues could be explained by either or both of the following: a novel function of the $2 b$ protein, which enables virus to spread into nonvascular cells, or the counter-defensive activities of the $2 \mathrm{~b}$ protein against basal host resistances (Lewsey et al. 2010).

The $2 \mathrm{~b}$ has been shown to have crucial roles in host-specific long-distance movement and in synergism caused by some CMV/Potyvirus spp. combinations. Ding and associates (1995) reported that $2 \mathrm{~b}$ has a function in determining systemic infectivity in cucumber. Wang and associates (2004) suggested that $2 \mathrm{~b}$ is essential for the CMV/Zucchini yellow mosaic virus synergism in squash. All these proposed functions do not fit our observations. The differences in the results are more likely due to differences in host responses or virus strains. Thus, we believe that there is a new mechanism for unloading of virus from vasculature by the $2 \mathrm{~b}$ protein.

\section{Local interference between two viruses can be detected even in the synergism between CMV and TuMV.}

The synergism between CMV and TuMV has been characterized by increases in symptom severity accompanied by cooperative support of TuMV accumulation or spread of CMV in the host plants (Fukuzawa et al. 2010; Sano and Kojima 1989; Takeshita and Takanami 2000). However, we discovered that A1Ds (or H1Ds) and TuEG actually competed with each other for their local infection sites in $N$. benthamiana, although their relationship was clearly synergistic. This indicated that, to receive a benefit from the synergistic interactions at an initial infection stage, CMV does not preferentially invade into the area which has been infected with TuMV. Similarly extensive spread of TuMV in the inoculated leaves was impeded within areas in which CMV infection has been established. The spa- tial antagonism caused a delay of systemic infection of A1DS after TuEG and that of TuEG after A1Ds. In the simultaneous A1Ds (H1Ds)/TuEG infection, unlike CMV, the systemic movement of TuMV seemed not to be affected. On the other hand, in the consecutive A1Ds-TuEG infection, the TuMV movement was actually delayed. Thus, we conclude that local interference actually decreased the viral accumulation levels in the inoculated leaves, which apparently affected viral spread but perhaps indirectly. CMV may require a relatively high level of virus accumulation for efficient systemic movement, whereas TuMV could move as efficiently as CMV, even with a low level of accumulation. This could have some relevance to the differences between the accumulation levels of CMV and those of TuMV in their host plants (Gal-On et al. 2000; Hammond and Lawson 1988).

The interference between CMV and TuMV might be due to host-induced antiviral responses, because the phenomenon was exacerbated by serial inoculation with one virus first and then the other. Whereas one virus might not induce certain host responses, or might suppress them, co-infection with the other virus led to these responses being activated then affecting the other virus. Whitham and associates (2003) found changes in Arabidopsis gene expression during a time course of infection against diverse plant RNA viruses, including CMV and TuMV. Thus, CMV and TuMV must each induce a separate series of differential host genes which are associated with plant stress or defense responses.

Interestingly, Mascia and associates (2010) reported that a decrease in accumulation of PVY in CMV/PVY synergism was due to the $2 \mathrm{~b}$. Similarly, the observation that TuMV decreased in the A1Ds/TuMV synergism also showed the nature of the competitive interactions between the two viruses in the synergism. We named this phenomenon local interference, which might be different from general interference called cross-protection in at least two points. First, the local interference was observed not only between two recombinant CMV, A1Ds and A1EG (or H1Ds and H1EG), but also between two different viruses, CMV and TuMV. Cross-protection is considered to be a phenomenon between two closely related viruses, in which RNA silencing has been implicated (Gal-On and Shiboleth 2006; Hull 2002; Ratcliff et al. 1999). Second, RNA silencing did not seem to play a major role in the local interference, because neither the strong RSS, TuMV HC-Pro, nor the low-temperature treatment to diminish RNA silencing affected the virus distribution; in fact, it was associated with an opposite phenomenon, synergism. However, siRNA analysis is necessary to rule out the involvement of silencing suppression in the mechanisms of the local interference. Takeshita and associates (2004a) showed that subgroups IA and II of CMV were largely too different in sequence to explain cross-protection between them by only RNA silencing. In agreement with our finding, Ziebell and associates (2007) and Ziebell and Carr (2009) previously reported that even general interference (cross-protection) was not fully explained by RNA silencing but might be considered as simple competition between two closely related viruses. Furthermore, Kurihara and Watanabe (2006) showed that cross-protection in Arabidopsis by two Tobamovirus spp. did not require RDR6, suggesting that RNA silencing does not play a major role in cross-protection. Taken together, based on the analyses of spatial infection patterns, we conclude that viral synergy and interference are not always antagonistic and not fully dependent on host RNA silencing.

Hammond and associates (1999) and Syller (2011) described different types of virus-virus interactions in mixed infections and suggested the biological significance of those complicated virus-virus interactions. Although the mechanisms involved in the local interference were masked by the synergism within 
the short period, it is conceivable that the phenomenon might play a pivotal role in the antagonistic virus-virus interactions of Hammond and associates (1999) and Syller (2011). Our novel findings provide a basis to understand CMV infection dynamics in association with antiviral host responses, interference (cross-protection), and synergism between viruses. We believe that the key step to reach this goal must be to elucidate all the roles of the multifunctional $2 b$ protein in CMV infection.

\section{MATERIALS AND METHODS}

\section{Plants and growth conditions.}

$N$. benthamiana plants were grown in an air-conditioned greenhouse at 25 and $20^{\circ} \mathrm{C}$ (day and night, respectively). In the examination at different temperature, inoculated plants were maintained under constant conditions in growth chambers at 25 and $20^{\circ} \mathrm{C}$ (day and night, respectively) or $15^{\circ} \mathrm{C}$ with a 14-h photoperiod.

\section{RNA extraction and tissue-blot hybridization.}

Total plant RNA extraction and tissue-blot hybridization analysis were performed as described previously (Takeshita et al. 2004a,b). Total RNA was extracted from plant tissue using TriZol reagent (Invitrogen, Carlsbad, CA, U.S.A.) essentially according to the supplied user protocol.

\section{Construction of modified CMV RNA2 vectors.}

Plasmid pCY2 was described previously (Suzuki et al. 1991). CMV RNA2 vectors (C2-A1 and C2-H1) derived from pCY2 were used for construction of the CMV RNA2 modified vectors. The DsRed 2 gene or the EGFP gene was introduced into the $\mathrm{C} 2-\mathrm{A} 1$ plasmid as detailed in Figure 1. All the primers used are listed in Supplementary Table 1. The DsRed2 ORF was PCR amplified from plasmid pDsRed2-1 (Clontech, Palo Alto, CA, U.S.A) using primers C2-A1-DsRed2-F and C2-A1DsRed2-R. The EGFP ORF was PCR amplified from plasmid pEGFP-1 (Clontech) using primers C2-A1-EGFP-F and C2A1-EGFP-R. All PCR assays were carried out with KOD-PlusDNA polymerase (TOYOBO, Osaka, Japan) using the following conditions: 30 cycles at $94^{\circ} \mathrm{C}$ for $30 \mathrm{~s}$, annealing at $50^{\circ} \mathrm{C}$ for $30 \mathrm{~s}$, and extension at $68^{\circ} \mathrm{C}$ for $60 \mathrm{~s}$. The PCR products were digested with $N r u \mathrm{I}$ and $M l u \mathrm{I}$, and $S t u \mathrm{I}$ and $M l u \mathrm{I}$, respectively. These fragments were cloned into StuI-MluI-digested C2-A1 to generate plasmids C2-A1-DsRed2 and C2-A1-EGFP, respectively (Fig. 1). The DsRed2 and EGFP genes also were introduced into the $\mathrm{C} 2-\mathrm{H} 1$ plasmid as illustrated in Figure 1. Primers C2-H1-DsRed2-F and C2-H1-DsRed2-R were applied to create $\mathrm{C} 2-\mathrm{H} 1-\mathrm{DsRed}$. Other primers (C2-H1-EGFP-F and C2-H1-EGFP-R) were used to construct C2-H1-EGFP. To generate a CMV vector for silencing of NbPDS gene expression, we used a highly homologous tomato PDS gene $(1,023$ to 1,124 for accession number EF650011). A 100-bp (1,023 to $1,124)$ tomato PDS (TmPDS) gene fragment was PCR amplified using the primers C2-A1-TmPDS-F and C2-A1-TmPDSR. The TmPDS gene fragments was cloned into StuI/MluIdigested C2-A1 to produce C2-A1-PDS (Fig. 1). All plasmid clones were verified by sequencing.

\section{Generation of wild-type, EGFP-, or}

DsRed2-expressing CMV viruses, and inoculation assay.

Plasmids pCY1, pCY2, pCY3 (containing full-length cDNA clone to CMV RNAs 1, 2, and 3 from strain Y), C2-A1, and $\mathrm{C} 2-\mathrm{H} 1$ and the newly constructed CMV vectors were used as templates for in vitro transcription as described previously (Suzuki et al. 1991). Wild-type CMV-Y was generated by inoculation of a mixture of in vitro transcripts derived from pCY1, pCY2, and pCY3 digested by NotI. CMV-Y derived from in vitro transcripts of the clones was propagated and purified as described previously by Takanami (1981). Unless otherwise specified, transcripts synthesized from plasmid pCY1 and pCY3 containing a full-length infectious RNA1 and RNA3 cDNA were used along with transcripts from recombinant plasmids derived from pCY2 in all inoculations. Yield and quality of the transcripts in the reaction mixture were analyzed on a $1.5 \%$ agarose gel. RNA transcripts (a mixture of RNA1, RNA2, and RNA3 transcripts) were used to inoculate leaves of $N$. benthamiana by rubbing. Full-length cDNA clone of wild-type TuMV (pTuR1) has been described by Suehiro and associates (2004). Wild-type TuMV and EGFP-expressing TuMV virus, named TuEG, was generated by bombardment inoculation of the plasmid clone to a leaf of $N$. benthamiana. Sap of the leaf tissues of $N$. benthamiana infected with TuMV or TuEG was used for mechanical inoculation assays. For simultaneous inoculation assays, the sap from a TuMV- or TuEG-infected leaf tissue was inoculated onto $N$. benthamiana immediately after inoculation of in vitro transcripts from the plasmid clones of CMV. Likewise, each inoculum was used for serial inoculation assays. For each inoculum, a set of three plants was used in symptom observation. The inoculation assays were repeated.

\section{Construction of $\mathbf{2 b}$ GFP-expressing transgenic plant.}

The $2 \mathrm{~b}$ coding sequence of CMV-Y was C-terminally fused with synthetic GFP coding sequence (Sheen et al. 1995). The PCR-amplified $2 \mathrm{~b}$ fragment was obtained using primers $2 \mathrm{~b}$ GFP-F and 2b-GFP-R. The GFP fragment was amplified with primers GFP-F and GFP-R. Both the fragments were ligated after digestion with BamHI. The resulting 2b-GFP fragment has a Gly-Ser-Gly-Gly-Gly spacer amino acid sequence between the $2 b$ and GFP ORF. The 2b-GFP fragment was cloned between the $X b a \mathrm{I}$ and $S a c \mathrm{I}$ sites of pBI121. The 2b-GFP vector was used for transformation. The construct was introduced into Agrobacterium tumefaciens by electroporation and transformed into leaf disks of $N$. benthamiana. The transformants were regenerated on Murashige-Skoog medium using kanamycin selection. Rooted plantlets were transferred to soil pots and grown in the greenhouse at $25^{\circ} \mathrm{C}$. Several lines with $2 \mathrm{~b}$ GFP construct were screened and regenerated. All the selected lines were homozygous and produced adequate amounts of seed for propagation.

\section{RT qPCR analysis.}

Total RNA samples were extracted from three biological replicates for validation of gene expression by comparative quantification assays. The first-strand cDNA was synthesized using $1.0 \mu \mathrm{g}$ of DNase I-treated RNA, oligo (dT) primer, random hexamer primer, and PrimeScript First-Strand cDNA Synthesis Kit (TaKaRa, Shiga, Japan). Comparative qPCR assays were performed using the Full Velocity SYBR Green QPCR Master Mix (Stratagene) on Real-Time PCR System (Stratagene, La Jolla, CA, U.S.A.). Each reaction contained $8.0 \mu$ l of the reaction mix, $0.24 \mu \mathrm{l}$ of Rox Reference dye, $0.16 \mu \mathrm{l}$ of 10 pmol each forward and reverse primer, $1.0 \mu \mathrm{l}$ of cDNA template (previously diluted 1:2), and water to $16 \mu$ l. Primers for DsREd2 ORF (qPCR-DsRed2-F and qPCR-DsRed2-R), coat protein (CP) ORF of TuMV (qPCR-TuMV CP-F and qPCR-TuMV CP-R), and the EFl $\alpha$ gene (qPCR-NtEF1 $\alpha-\mathrm{F}$ and qPCR-NtEF1 $\alpha-\mathrm{R}$ ) were used for qPCR. Samples were incubated at $50^{\circ} \mathrm{C}$ for $5 \mathrm{~min}$, followed by 40 cycles of $95^{\circ} \mathrm{C}$ for $30 \mathrm{~s}$ and $60^{\circ} \mathrm{C}$ for $30 \mathrm{~s}$. Relative quantification was performed using the standard curve method, and transcript accumulation of each gene was normalized to the quantity of $E F l \alpha$. The fold change for each zone was calculated by dividing the relative expression level of the A1Ds(or A1Ds/TuEG)-infected sample by the corresponding A1Ds/ 
TuEG- (or TuEG)-inoculated sample. These values were transformed and plotted with standard errors in Figure 5. The data were obtained from each assay, which were repeated.

\section{Semiquantitative RT-PCR.}

First-strand cDNAs of viral RNA and mRNA were synthesized using extracted total plant RNA according to the procedure described by Takeshita and associates (2009). The target regions in the cDNAs were amplified by PCR from 1- $\mu$ l aliquots of the cDNA reaction mixture with CMV RNA3-specific primers, the HC-Pro gene of TuMV-targeting primers, and a part of the $E F 1 \alpha$ gene-specific primers. The process of thermocycling reactions using a primer set and KOD-Plus-DNA polymerase (TOYOBO) was as follows: first step $\left(94^{\circ} \mathrm{C}\right.$ for $\left.2 \mathrm{~min}\right)$ and second step with 10 to 45 cycles $\left(94^{\circ} \mathrm{C}\right.$ for $30 \mathrm{~s}, 50^{\circ} \mathrm{C}$ for $30 \mathrm{~s}$, and $68^{\circ} \mathrm{C}$ for $2 \mathrm{~min}$ ). Aliquots containing $20 \mu \mathrm{l}$ of the PCR mixture were prepared for each sample in nine or eight PCR tubes. All the tubes were examined in the same PCR reaction. One tube for each sample was removed at every five PCR cycle between 10 to 40 or 10 to 45 cycles. Primers for CMV (semi-qPCR-CMVR) $3^{\prime}$ terminus of the HC-Pro gene of TuMV ( $3^{\prime}$ terminus TuMV HC-Pro), and the EFl $\alpha$ gene (oligo dT18) were used for reverse transcription. Primers for CMV RNA3 (semi-qPCRCMV-F and semi-qPCR-CMV-R), HC-Pro ORF of TuMV (semi-qPCR-TuMV-F and semi-qPCR-TuMV-R), and the EFl $\alpha$ gene (semi-qPCR-NtEF1 $\alpha-\mathrm{F}$ and semi-qPCR-NtEF1 $\alpha-\mathrm{R}$ ) were used for PCR. The primers were also used for CMV- and TuMV-specific detection by RT-PCR. For each inoculum, a set of three plants was used. The semiquantitative RT-PCR assays were repeated using the samples from the independent inoculation tests to confirm the results.

\section{Nucleotide sequencing.}

Sequencing was done using the Big Dye Terminator DNA Sequencing Kit (version 3.1; Applied Biosystems, Foster City, CA, U.S.A.) and the ABI Prism 310 genetic analyzer. Sequence analysis was performed using the program GENETYX-Win (version 10; Genetyx Corporation, Tokyo).

\section{Protoplast assay for RNA-silencing suppressors.}

Protoplasts were prepared from leaves of $N$. benthamiana as described by Shimura and associates (2008). The protoplast assay was performed essentially according to the protocol in Shimura and associates (2008) and Asaoka and associates (2010). Firefly luciferase (Fluc) was used as the target of RNA silencing and Renilla luciferase was done as the internal control for transfection. As a silencing inducer, dsRNA of the Fluc gene (dsFluc) was introduced into the protoplasts. Viral suppressor proteins ( $2 \mathrm{~b}$ and $\mathrm{P} 19)$ were expressed in protoplasts from RNA transcripts, which were prepared from the PCRamplified RNA4A fragment of CMV. The results were obtained from three replicates. The assays were repeated twice.

\section{Fluorescence microscopy.}

Green and red fluorescence images of leaf tissues were acquired using SMZ1500 (Nikon, Tokyo) with GFP and DsRed filter sets. Adobe Photoshop was used for acquiring digital images, which were exported as JPEG files. For each inoculum, a set of three plants was used. Leaves of different plants were removed and used for imaging at different days postinoculation. The data were obtained from each assay, which was repeated once or twice.

\section{ACKNOWLEDGMENTS}

We thank P. Palukaitis for critically reading this manuscript and his suggestions and D. C. Baulcome for providing the P19 gene. This work was funded, in part, by Grants-in-Aid for Scientific Research 14760029 and 18780030 from The Ministry of Education, Science, Sports and Culture, Japan.

\section{LITERATURE CITED}

Asaoka, R., Shimura, H., Arai, M., and Masuta, C. 2010. A progeny virus from a Cucumovirus pseudorecombinant evolved to gain the ability to accumulate its RNA-silencing suppressor leading to systemic infection in tobacco. Mol. Plant-Microbe Interact. 23:332-339.

Brigneti, G., Voinnet, O., Li, W.-X., Ji, L.-H., Ding, S.-W., and Baulcombe, D. C. 1998. Viral pathogenicity determinants are suppressors of transgene silencing in Nicotiana benthamiana. EMBO (Eur. Mol. Biol. Organ.) J. 17:6739-6746.

Ding, S. W., Anderson, B. J., Haase, H. R., and Symons, R. H. 1994. New overlapping gene encoded by the cucumber mosaic virus genome. $\mathrm{Vi}$ rology 198:593-601.

Ding, S. W., Li, W. X., and Symons, R. H. 1995. A novel naturally occurring hybrid gene encoded by a plant RNA virus facilitates long distance virus movement. EMBO (Eur. Mol. Biol. Organ.) J. 14:5762-5772.

Fukuzawa, N., Itchoda, N, Ishihara, T., Goto, K., Masuta, C., and Matsumura, T. 2010. HC-Pro, a Potyvirus RNA silencing suppressor, cancels cycling of Cucumber mosaic virus in Nicotiana benthamiana plants. Virus Genes 40:440-446.

Gal-On, A., and Shiboleth, Y. M. 2006. Cross-protection. Pages 261-288 in: Natural Resistance Mechanisms of Plants to Viruses. G. Loebenstein and J. P. Carr, eds. Springer Publishers, Drodrecht, The Netherlands.

Gal-On, A., Canto, T., and Palukaitis, P. 2000. Characterisation of genetically modified Cucumber mosaic virus expressing histidine-tagged 1a and 2a proteins. Arch. Virol. 145:37-50.

González, I., Martínez, L., Rakitina, D. V., Lewsey, M. G., Atencio, F. A., Llave, C., Kalinina, N. O., Carr, J. P., Palukaitis, P., and Canto, T. 2010. Cucumber mosaic virus $2 \mathrm{~b}$ protein subcellular targets and interactions: Their significance to RNA silencing suppressor activity. Mol. Plant-Microbe Interact. 23:294-303.

González-Jara, P., Tenllado, F., Martínez-García, B., Atencio, F. A., Barajas, D., Vargas, M., Díaz-Ruiz, J., and Díaz-Ruíz, J. R. 2004. Host-dependent differences during synergistic infection by potyviruses with Potato virus X. Mol. Plant Pathol. 5:29-35.

Goto, K., Kobori, T., Kosaka, Y., Natsuaki, T., and Masuta, C. 2007. Characterization of silencing suppressor $2 \mathrm{~b}$ of Cucumber mosaic virus based on examination of its small RNA-binding abilities. Plant Cell Physiol. 48:1050-1060.

Hammond J., and Lawson, R. H. 1988. An improved purification procedure for preparing potyviruses and cytoplasmic inclusions from the same tissue. J. Virol. Methods 20:203-217.

Hammond, J., Lecoq, H., and Raccah, B. 1999. Epidemiological risks from mixed virus infections and transgenic plants expressing viral genes. Adv. Virus. Res. 54:189-314.

Hull, R. 2002. Matthews' Plant Virology, 4th ed. Academic Press, New York.

Ji, L. H., and Ding, S. W. 2001. The suppressor of transgene RNA silencing encoded by Cucumber mosaic virus interferes with salicylic acidmediated virus resistance. Mol. Plant-Microbe Interact. 14:715-724.

Kanazawa, A., Inaba, J., Shimura, H., Otagaki, S., Tsukahara, S., Matsuzawa, A., Kim, B. M., Goto, K., and Masuta, C. 2011. Virus-mediated efficient induction of epigenetic modifications of endogenous genes with phenotypic changes in plants. Plant J. 65:156-168.

Kurihara, Y., and Watanabe, Y. 2006. Cross-protection in Arabidopsis against crucifer tobamovirus $\mathrm{Cg}$ by an attenuated strain of the virus. Mol. Plant Pathol. 4:259-269.

Lewsey, M. G., Murphy, A. M., MacLean, D., Dalchau, N., Westwood, J. H., Macaulay, K., Bennett, M. H., Moulin, M., Hanke, D. E., Powell, G., Smith, A. G., and Carr, J. P. 2010. Disruption of two defensive signaling pathways by a viral RNA silencing suppressor. Mol. Plant-Microbe Interact. 23:835-845.

Mascia, T., Cillo, F., Fanelli, V., Finetti-Sialer, M. M., De Stradis, A., Palukaitis, P., and Gallitelli, D. 2010. Characterization of the interactions between Cucumber mosaic virus and Potato virus $Y$ in mixed infections in tomato. Mol. Plant-Microbe Interact. 23:1514-1524.

Matsuo, K., Hong, J.-S., Tabayashi, N., Ito, A., Masuta, C., and Matsumura, T. 2007. Development of Cucumber mosaic virus as a vector modifiable for different host species to produce therapeutic proteins. Planta 225:277286.

Otagaki, S., Arai, M., Takahashi, A., Goto, K., Hong, J.-S., Masuta, C., and Kanazawa, A. 2006. Rapid induction of transcriptional and posttranscriptional gene silencing using a novel Cucumber mosaic virus vector. Plant Biotech. 23:259-265.

Palukaitis, P., and García-Arenal, F., 2003. Cucumoviruses. Adv. Virus. Res. 62:241-323. 
Ratcliff, F. G., MacFarlane, S. A., and Baulcombe, D. C. 1999. Gene silencing without DNA. RNA-mediated cross protection between viruses. Plant Cell 11:1207-1216.

Ryang, B.-S., Kobori, T., Matsumoto, T., Kosaka, Y., and Ohki, S. T. 2004 Cucumber mosaic virus $2 \mathrm{~b}$ protein compensates for restricted systemic spread of Potato virus $Y$ in doubly infected tobacco. J. Gen. Virol. 85:3405-3414.

Ryang, B.-S., Matsumoto, T., Kobori, T., Kosaka, Y., and Ohki, S. T. 2005. $2 \mathrm{~b}$ Protein is essential to induce a novel gradual cell death in Zucchini yellow mosaic virus-inoculated cucumber cotyledon co-infected with Cucumber mosaic virus. J. Gen. Plant Pathol. 71:308-313.

Sano, Y., and Kojima, M. 1989. Increase in cucumber mosaic virus concentration in Japanese radish plants co-infected with turnip mosaic virus. Ann. Phytopathol. Soc. Jpn. 55:296-302.

Sheen, J., Hwang, S., Niwa, Y., Kobayashi, H., and Galbraith, D. W. 1995. Green-fluorescent protein as a new vital marker in plant cells. Plant J. 8:777-784.

Shi, B. J., Miller, J., Symons, R. H., and Palukaitis, P. 2003. The 2b protein of cucumoviruses has a role in promoting the cell-to-cell movement of pseudorecombinant viruses. Mol. Plant-Microbe Interact. 16:261-267.

Shimura, H., Fukagawa, T., Meguro, A., Yamada, H., Oh-hira, M., Sano, S., and Masuta, C. 2008. A strategy for screening an inhibitor of viral silencing suppressors, which attenuates symptom development of plant viruses. FEBS (Fed. Eur. Biochem. Soc.) Lett. 582:4047-4052.

Soards, A. J., Murphy, A. M., Palukaitis, P., and Carr, J. P. 2002. Virulence and differential local and systemic spread of Cucumber mosaic virus in tobacco are affected by the CMV 2b protein. Mol. Plant-Microbe Interact. 15:647-653.

Suehiro, N., Natsuaki, T., Watanabe T., and Okuda, S. 2004. An important determinant of the ability of Turnip mosaic virus to infect Brassica spp. and/or Raphanus sativus is in its P3 protein. J. Gen. Virol. 85:20872098.

Suzuki, M., Kuwata, S., Kataoka, J., Masuta, C., Nitta, N., and Takanami, Y. 1991. Functional analysis of deletion mutants of cucumber mosaic virus RNA3 using an in vitro transcription system. Virology 183:106-113.

Syller, J. 2011. Facilitative and antagonistic interactions between plant viruses in mixed infections. Mol. Plant Pathol. Published online. doi: 10.1111/J.1364-3703.2011.00734.X.

Szittya, G., Silhavy, D., Molnár, A., Havelda, Z., Lovas, Á., Lakatos, L., Bánfalvi, Z., and Burgyán, J. 2003. Low temperature inhibits RNA si- lencing-mediated defence by the control of siRNA generation. EMBO (Eur. Mol. Biol. Organ.) J. 22:633-640.

Takanami, Y., 1981. A striking change in symptoms on cucumber mosaic virus-infected tobacco plants induced by a satellite RNA. Virology 109:120-126.

Takeshita, M., and Takanami, Y. 2000. Defective long-distance transport of Cucumber mosaic virus in radish is efficiently complemented by Turnip mosaic virus. J. Gen. Plant Pathol. 66:254-257.

Takeshita, M., Kikuhara, K., Kuwata, S., Furuya, N., and Takanami, Y. 2004a. Competition between wild-type virus and a reassortant from subgroups I and II of CMV and activation of antiviral responses in cowpea. Arch. Virol. 149:1851-1857.

Takeshita, M., Shigemune, N., Kikuhara, K., Furuya, N., and Takanami, Y. 2004b. Spatial analysis for exclusive interactions between subgroups I and II of Cucumber mosaic virus in cowpea. Virology 328:45-51.

Takeshita, M., Matsuo, Y., Suzuki, M., Furuya, N., Tsuchiya, K., and Takanami, Y. 2009. Impact of a defective RNA 3 from Cucumber mosaic virus on helper virus infection dynamics. Virology 389:59-65.

Voinnet, O., Pinto, Y. M., and Baulcombe, D. C. 1999. Suppression of gene silencing: A general strategy used by diverse DNA and RNA viruses of plants. Proc. Natl. Acad. Sci. U.S.A. 96:14147-14152.

Wang, Y., Tzfira, T., Gaba, V., Citovsky, V., Palukaitis, P., and Gal-On, A. 2004. Functional analysis of the Cucumber mosaic virus $2 \mathrm{~b}$ protein: Pathogenicity and nuclear localization. J. Gen. Virol. 85:3135-3147.

Whitham, S. A. , Quan, S., Chang, H.-S., Cooper, B., Estes, B., Zhu, T. Wang, X., and Hou, Y.-H. 2003. Diverse RNA viruses elicit the expression of common sets of genes in susceptible Arabidopsis thaliana plants. Plant J. 33:271-283.

Ziebell, H., and Carr, J. P. 2009. Effects of dicer-like endoribonucleases 2 and 4 on infection of Arabidopsis thaliana by Cucumber mosaic virus and a mutant virus lacking the $2 \mathrm{~b}$ counter-defence protein gene. J. Gen. Virol. 90:2288-2292.

Zhang, X., Yuan, Y.-R., Pei, Y., Lin, S.-S., Tuschl, T., Patel, D. J., and Chua, N.-H. 2006. Cucumber mosaic virus-encoded 2b suppressor inhibits Arabidopsis Argonaute1 cleavage activity to counter plant defense. Genes Dev. 20:3255-3268.

Ziebell, H., Payne, T., Berry, J. O., Walsh, J. A., and Carr, J. P. 2007. A Cucumber mosaic virus mutant lacking the $2 \mathrm{~b}$ counter-defence protein gene provides protection against wild-type strains. J. Gen. Virol. $88: 2862-2871$ 